\title{
Chemical Constituents of the Methanolic Extract of Leaves of Leiothrix spiralis Ruhland and Their Antimicrobial Activity
}

Marcelo Gonzaga de Freitas Araújo ${ }^{1}$, Felipe Hilário ${ }^{2}$, Leonardo Gorla Nogueira ${ }^{\mathbf{1}}$, Wagner Vilegas ${ }^{2}$, Lourdes Campaner dos Santos ${ }^{2}$ and Taís Maria Bauab ${ }^{1, *}$

1 Department of Biological Sciences, Faculty of Pharmaceutical Sciences, São Paulo State University-UNESP, 14801-902, Araraquara, SP, Brazil

2 Department of Organic Chemistry, Chemistry Institute, São Paulo State University-UNESP, 14800-900, Araraquara, SP, Brazil

* Author to whom correspondence should be addressed; E-Mail: bauabtm@fcfar.unesp.br; Tel.: +55-16-3301-6955; Fax: +55-16-3301-6940.

Received: 1 November 2011; in revised form: 1 December 2011 / Accepted: 5 December 2011 / Published: 16 December 2011

\begin{abstract}
Chemical fractionation of the methanolic extract of leaves of Leiothrix spiralis Ruhland afforded the flavonoids luteolin-6-C- $\beta$-D-glucopyranoside (1), 7-methoxyluteolin6-C- $\beta$-D-glucopyranoside (2), 7-methoxyluteolin-8- $C$ - $\beta$-D-glucopyranoside 4'-methoxyluteolin-6-C- $\beta$-D-glucopyranoside (4), and 6-hydroxy-7-methoxyluteolin (5), and the xanthones 8-carboxymethyl-1,5,6-trihydroxy-3-methoxyxanthone (6), 8-carboxymethyl-1,3,5,6-tetrahydroxyxanthone (7). Methanolic extract, fractions, and isolated compounds of the leaves of $L$. spiralis were assayed against Gram-positive (Staphylococcus aureus, Bacillus subtilis and Enterococcus faecalis) and Gram-negative bacteria (Escherichia coli, Pseudomonas aeruginosa, Salmonella setubal and Helicobacter pylori) and fungi (the yeasts Candida albicans, C. tropicalis, C. krusei and C. parapsilosis). We observed the best minimum inhibitory concentration values for the methanolic extract against Candida parapsilosis, for the fraction $5+6$ against Gramnegative bacteria E. coli and P. aeruginosa, and compound 7 against all tested Candida strains. The methanolic extract contents suggest that this species may be a promising source of compounds to produce natural phytomedicines.
\end{abstract}

Keywords: Eriocaulaceae; Leiothrix spiralis; phenolic compounds; antimicrobial activity 


\section{Introduction}

The Eriocaulaceae is a pantropical family which consists of 11 genera and about 1,200 species. The genus Leiothrix belongs to the Eriocaulaceae family and is exclusively South American, including about 37 species mainly restricted to Brazil [1]. All species of Leiothrix subg. Stephanophyllum are pseudoviviparous, and some of them (L. spiralis and L. vivipara) are endemic to the state of Minas Gerais, Brazil. This specie is a frequent component of the vegetation in pools of hilly areas or swamps, especially in sandy ground areas, with low water retention [2]. Little is known about the ethnopharmacological property of Eriocaulaceae. Literature reports the traditional use of Eriocaulaceae species to treat skin ulcers and bacterial infections [3], and some studies have described the antioxidant [4], cytotoxic, mutagenic [5,6], and antiulcerogenic activities [7]. Meanwhile, some species of this family are of great economic importance since they are exported as ornamental plants to various countries, mainly Germany and Japan [8].

Chemical studies on Leiothrix species are also scarce. The presence of soluble phenolic compounds with antimicrobial activity has been investigated in ramets of 21 Leiothrix species, with identification of nepetin-7-O- $\beta$-D-glucopyranoside, nepetin-7- $O$ - $\beta$-D-arabinopyranoside, and luteolin $O$ - and $C$-glucopyranoside $[9,10]$. Another study has determined the presence of xanthones and flavone with antioxidant activity in ramets/flower heads of L. flavescens and L. curvifolia [11]. Therefore, we performed a phytochemical study of methanolic extract of the L. spiralis leaves and investigated the antimicrobial activity of the extract, fractions and isolated compounds on different strains of bacteria and yeasts.

\section{Results and Discussion}

The structures of isolated compounds 1-7 were determined by spectroscopic analysis (1D-, 2D-NMR, UV, ES-MS) and by comparison of spectral data to related metabolites (Figure 1). The chemical constituents of Eriocaulaceae are of taxonomic importance. Naphthopyranones and flavonols are the major compounds in many Paepalanthus species [9,12], but are absent in species belonging to both the Syngonanthus and the Leiothrix genus [2,9,13,14].

Figure 1. Compounds isolated from the $\mathrm{MeOH}$ extract of leaves of L. spiralis.

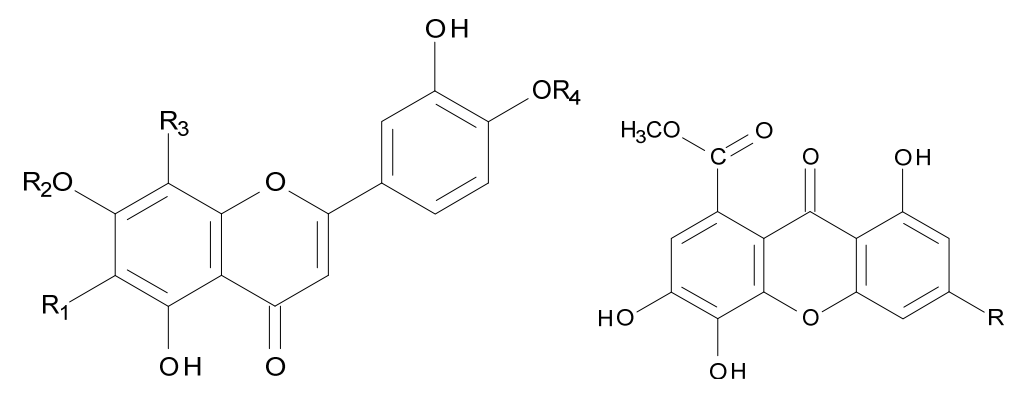

\begin{tabular}{cllllcl}
\hline Comp. & $\mathbf{R}_{\mathbf{1}}$ & $\mathbf{R}_{\mathbf{2}}$ & $\mathbf{R}_{\mathbf{3}}$ & $\mathbf{R}_{\mathbf{4}}$ & Comp. & $\mathbf{R}$ \\
\hline $\mathbf{1}$ & $C$-Glc & $\mathrm{H}$ & $\mathrm{H}$ & $\mathrm{H}$ & $\mathbf{6}$ & $\mathrm{OH}$ \\
$\mathbf{2}$ & $C$-Glc & $\mathrm{CH}_{3}$ & $\mathrm{H}$ & $\mathrm{H}$ & 7 & $\mathrm{OCH}_{3}$ \\
$\mathbf{3}$ & $\mathrm{H}$ & $\mathrm{CH}_{3}$ & $C$-Glc & $\mathrm{H}$ & & \\
$\mathbf{4}$ & $C-\mathrm{Glc}$ & $\mathrm{H}$ & $\mathrm{H}$ & $\mathrm{CH}_{3}$ & & \\
$\mathbf{5}$ & $\mathrm{OH}$ & $\mathrm{CH}_{3}$ & $\mathrm{H}$ & $\mathrm{H}$ & & \\
\hline
\end{tabular}


In this study, five flavone and two xanthones were detected in a methanolic extract of L. spiralis. The presence of flavones in L. spiralis corroborates the previously reported data [9]. Xanthones 6 and 7 have been isolated from L. curvifolia and L. flavescens [7,13] and present antioxidant activity [11]. The presence of a $\mathrm{COOCH}_{3}$ group at $\mathrm{C}-8$ of the xanthone ring is unusual, since most xanthones isolated from higher plants presents a methyl group at $\mathrm{C}-3$, while the xanthones isolated from lichens have a methyl group at C-8 position [15]. Previous investigators have proposed that lichen xanthones are biosynthesized by cyclization of a single, linear polyketide chain [16]. It has been proposed that Leiothrix xanthones are biosynthetically related to naphthopyranones isolated from species of the Paepalanthus genus (Eriocaulaceae) [12].

Minimum inhibitory concentration (MIC) analysis revealed antibacterial and antifungal activity of the almost all tested sample (Table 1). Holetz et al. [17] considered that if the extracts displayed an MIC less than $100 \mu \mathrm{g} / \mathrm{mL}$, the antimicrobial activity was good; from 100 to $500 \mu \mathrm{g} / \mathrm{mL}$ the antimicrobial activity was moderate; from 500 to $1,000 \mu \mathrm{g} / \mathrm{mL}$ the antimicrobial activity was weak; over $1,000 \mu \mathrm{g} / \mathrm{mL}$ the extract was considered inactive. Based on these criteria, the extract presented described that MIC concentrations higher than $100 \mu \mathrm{g} / \mathrm{mL}$ of phytochemical weak activity against Gram-positive bacteria, and moderate activity against $C$. parapsilosis. Gibbons [18] compounds would not be expected to have clinical significance. MIC results show that Fraction $\mathbf{5}+\mathbf{6}$ and compound $\mathbf{7}$ were able to prevent the growth of some tested bacterial species. The fraction $\mathbf{5}+\mathbf{6}$ showed the lowest MIC value against $E$. coli $(62.5 \mu \mathrm{g} / \mathrm{mL})$ and $P$. aeruginosa $(31.25 \mu \mathrm{g} / \mathrm{mL})$. This activity could be considered as promising, considering the fact that the tested bacteria are resistant to first line antibiotics, and also that $P$. aeruginosa is a leading cause of nosocomial infections $[19,20]$.

Determination of minimum bacterial concentration (MBC) and minimum fungicidal concentration (MFC) (Table 1) confirmed that a cidal effect of most of the tested samples could be expected on the studied microorganisms. As can be observed, most of the significant MBC and MFC values are similar to the corresponding MIC values, mainly in yeasts.

All tested samples were found to be active against at least one of Candida species. Compound 7 had the best result against all tested yeast species. Previous studies have reported some xanthone derivatives as remarkable antifungal agents. The antifungal profile of the described xanthones suggests that, in the majority of cases, hydroxyl groups are important for activity [21,22]. It was reported as likely that antifungal xanthones from plants required three or four hydroxyl groups, in which one or two of them must be at C-5 and/or C-6, and a hydrophobic group must be on one of the aromatic rings, and compound 7 meets these requirements [23]. We demonstrated that compound 7 exhibits fungicidal activity against $C$. krusei and C. parapsilosis. According to the literature, in general, xanthones have fungicidal rather than fungistatic activity. The putative mechanism pointed to the cell membrane as a possible target, by hypothesizing that xanthones act by the inhibition of ergosterol biosynthesis [24]. The antifungal activity of the phenolic compounds has been attributed to their lipophilic properties, which determine their ability to penetrate into the plasma membrane and induce changes in the physico-chemical properties of the cell wall, cell membrane, and cellular organelles. Another hypothesis include inhibition of enzimes produced by fungi and authors have linked the antioxidant activity of phenolic compounds to their activity on the biosynthesis inhibition of these metabolites [25]. The strong in-vitro antifungal activity of xanthone suggests that this compound might have wide pharmaceutical use. 
Table 1. Antibacterial and antifungal activity of methanolic extract, fractions, and compounds isolated from L. spiralis leaves.

\begin{tabular}{|c|c|c|c|c|c|c|c|c|c|c|}
\hline \multirow{3}{*}{ Tested samples } & \multicolumn{6}{|c|}{ MIC (MBC) $)^{\mathrm{a}}$} & \multicolumn{4}{|c|}{ MIC (MFC) ${ }^{\mathrm{a}}$} \\
\hline & \multicolumn{3}{|c|}{ Gram-positive bacteria } & \multicolumn{3}{|c|}{ Gram-negative bacteria } & \multicolumn{4}{|c|}{ Fungi } \\
\hline & S. aureus & B. subtilis & E. faecalis & E. coli & P. aeruginosa & S. setubal & C. albicans & C. krusei & C. parapsilosis & C. tropicalis \\
\hline Extract & $1000(1000)$ & $1000(1000)$ & $500(-)$ & - & - & - & $1000(1000)$ & $500(1000)$ & $250(250)$ & $1000(1000)$ \\
\hline Fraction $(5+6)$ & $125(250)$ & $250(250)$ & $-*$ & $62.5(250)$ & $31.25(250)$ & $-*$ & $-*$ & $-*$ & $125(125)$ & $-*$ \\
\hline Fraction $(\mathbf{2}+\mathbf{3})$ & $-*$ & $-*$ & $-*$ & $-*$ & $-*$ & $-*$ & $-*$ & $250(250)$ & $125(125)$ & $250(250)$ \\
\hline (1) & $-*$ & $-*$ & $-*$ & $-*$ & $-*$ & $-*$ & $-*$ & $-*$ & $125(125)$ & $-*$ \\
\hline (4) & $-*$ & $-*$ & $-*$ & $-*$ & $-*$ & $-*$ & $125(250)$ & $-*$ & $-*$ & $-*$ \\
\hline (7) & $125(-*)$ & $125(125)$ & $-*$ & $-*$ & $125(250)$ & $-*$ & $62.5(250)$ & $15.7(15.7)$ & $15.7(15.7)$ & $31.25(62.5)$ \\
\hline Positive controls ${ }^{b}$ & $\begin{array}{c}2.1 \times 10^{-2} \\
\left(2.1 \times 10^{-2}\right)\end{array}$ & $\begin{array}{c}2.1 \times 10^{-2} \\
\left(2.1 \times 10^{-2}\right)\end{array}$ & $\begin{array}{c}2.1 \times 10^{-2} \\
\left(2.1 \times 10^{-2}\right)\end{array}$ & $\begin{array}{c}3.3 \times 10^{-5} \\
\left(3.3 \times 10^{-5}\right)\end{array}$ & $\begin{array}{c}3.3 \times 10^{-5} \\
\left(3.3 \times 10^{-5}\right)\end{array}$ & $\begin{array}{c}3.3 \times 10^{-5} \\
\left(3.3 \times 10^{-5}\right)\end{array}$ & $32(>64)$ & $8(>64)$ & $8(>64)$ & $32(>64)$ \\
\hline
\end{tabular}

${ }^{a}$ Values given as $\mu \mathrm{g} / \mathrm{mL} .{ }^{b}$ ciprofloxacin for bacteria and fluconazole for fungi (-) MIC/MBC/MFC > $1000 \mu \mathrm{g} / \mathrm{mL} ;(-*) \mathrm{MIC} / \mathrm{MBC} / \mathrm{MFC}>500 \mu \mathrm{g} / \mathrm{mL}$. MIC: $\mathrm{minimum}$ inhibitory concentration; MBC/MFC: minimum bactericidal/fungicial concentration. 
All other samples had no significant activity. Despite of the phenolic content, the extract showed weak activity against Gram-positive bacteria. However, these findings suggest that phenols, mainly xanthones, are among the chemical classes responsible for the antibacterial activity of L. spiralis. Fang et al. [26] found that five phenols, among then xanthones and flavones, isolated from Eriocaulon buergerianum, a Eriocaulaceae specie, exhibited potential antibacterial activity against $S$. aureus. The flavones of Fraction $\mathbf{2}+\mathbf{3}$ showed moderate activity against Candida species. Previous investigators have also documented the antibacterial and antifungal properties of flavonoids [26-29]. This activity might be explained by their ability to complex with cell wall and induce the formation of pseudomulticellular aggregates, inhibiting microbial growth [30,31]. Methanolic extract of leaves of L. spiralis inhibited weakly the growth of all yeasts tested. Silva et al. [32] have reported the anti-C. albicans activity of this extract. The MIC values observed by us are similar to those reported by Silva et al. [32]; however, the activity was observed in the present study against other Candida species.

Activity against $H$. pylori of the extract and compounds tested was not evident under the present experimental conditions, and zones of inhibition were not produced. In this study, the bacteria was susceptible to clarithromycin (positive control), whose diameter of inhibition zone was $21.8 \pm 1 \mathrm{~mm}$. A previous study has shown anti-H. pylori activity of the methanolic extract of L. flavescens. Such activity can be correlated with the presence of flavones and xanthones with a catechol nucleus in their structures [33]. Despite the fact that most of the compounds detected in methanolic extract of leaves of $L$. spiralis were flavones and xanthones, we used a different methodology and did not observe activity against $H$. pylori.

\section{Experimental}

\subsection{General Procedures}

Melting point was measured on a MQ APF-301 (Microquímica ${ }^{\circledR}$, Brazil) digital apparatus. UV spectrum was recorded on a HACH UV-Vis DR/4000 spectrophotometer in $\mathrm{MeOH}$ [34]. IR spectrum was obtained using Shimadzu FT-IR 8300 spectrophotometer in KBr disk. NMR analyses and 2D experiments were run on Varian ${ }^{\circledR}$ INOVA 500 operating at $500 \mathrm{MHz}$ for ${ }^{1} \mathrm{H}$ and $125 \mathrm{MHz}$ for ${ }^{13} \mathrm{C}(11.7 \mathrm{~T})$, using TMS as internal standard. ESIMS in negative and positive ion mode and mass spectra were acquired and processed using the software (version 1.3) provided by the manufacturer. The capillary voltage was set at $-32 \mathrm{~V}$, the spray voltage at $5 \mathrm{kV}$ and the tube lens offset at $30 \mathrm{~V}$. Capillary temperature was $280{ }^{\circ} \mathrm{C}$. Each compound was dissolved in $\mathrm{CH}_{3} \mathrm{OH}$ and infused in the ESI source by using a syringe pump (flow rate $5 \mu \mathrm{L} / \mathrm{min}$ ). TLC analyses were performed on silica gel $200 \mu \mathrm{m}$ (Sorbent Technologies ${ }^{\circledR}$ ) and visualized using UV light (254 and $365 \mathrm{~nm}$ ).

\subsection{Plant Material}

Leaves of L. spiralis were collected in May 2006 in Diamantina, state of Minas Gerais, Brazil and authenticated by Dr. Paulo Takeo Sano from the Institute of Biosciences of the University of São Paulo (IB-USP), São Paulo. A voucher specimen (SANO No. 4798) was deposited at the IB-USP Herbarium. 


\subsection{Extraction and Isolation}

Leaves of $L$. spiralis were dried in an oven at $45{ }^{\circ} \mathrm{C}$ for one week. The dried leaves of $L$. spiralis $(2.00 \mathrm{~g})$ were powdered and extracted successively with hexane, methylene chloride (500 $\mathrm{mL}$ each solvent) by maceration at room temperature (one week each solvent) and the methanol $(500 \mathrm{~mL})$ were extracted by percolation (one week). The solvents were removed under vacuum to yield the crude extracts. The methanol extract $(1.2 \mathrm{~g})$ was chromatographed on a Sephadex LH-20 column $(80 \times 2 \mathrm{~cm})$, with $\mathrm{MeOH}$ as eluent.

Fractions $(8 \mathrm{~mL})$ were collected and checked by TLC [ $\mathrm{Si}$ gel plates, $\mathrm{CHCl}_{3} / \mathrm{MeOH} / n-\mathrm{PrOH} / \mathrm{H}_{2} \mathrm{O}$, $(5: 6: 1: 4, \mathrm{v} / \mathrm{v} / \mathrm{v} / \mathrm{v})]$. Fractions $\mathbf{7 9 - 8 2}(70 \mathrm{mg})$ were further purified by medium pressure liquid chromatography (MPLC) using a column of RP-18 silica gel $(9.0 \mathrm{~cm} \times 1.5 \mathrm{~mm}$ i.d.) eluted with $\mathrm{MeOH}$ $\mathrm{H}_{2} \mathrm{O}$ (5:95 v/v, initial, after gradient until methanol), affording 4'-methoxyluteolin-6-C- $\alpha$-Lglucopyranoside (4, 8.0 mg) [35], 8-carboxymethyl-1,3,5,6-tetrahydroxyxanthone (7, 7.0 mg), 8-carboxymethyl-1,5,6-trihydroxy-3-methoxyxanthone $(6,9.0 \mathrm{mg})$ [13], and 6-hydroxy-7-methoxyluteolin $(\mathbf{5}, 10.0 \mathrm{mg})$ [36]. Fractions 61-68 $(295 \mathrm{mg})$ were further purified by MPLC using the same conditions described for fractions 79-82, yielding 7-methoxyluteolin-6- $C$ - $\beta$-D-glucopyranoside $(\mathbf{2}, 12.0 \mathrm{mg})$ [37] and 7-methoxyluteolin-8-C- $\beta$-D-glucopyranoside $(\mathbf{3}, 15.0 \mathrm{mg})$ [38]. Fractions 69-74 $(30 \mathrm{mg}$ ) were further purified by MPLC using the same conditions indicated for fractions 79-82, yielding luteolin-6- $C$ - $\beta$-D-glucopyranoside $(1,10.0 \mathrm{mg}$ ) [39]. Although all the compounds have been identified separately, compounds $\mathbf{2}$ and $\mathbf{3}$ were isolated in a single fraction, as well as the compounds $\mathbf{5}$ and $\mathbf{6}$, thus named Fraction $(\mathbf{2}+\mathbf{3})$ and Fraction $(\mathbf{5}+\mathbf{6})$, respectively.

Luteolin-6-C- $\beta$-D-glucopyranoside (1): UV $\lambda_{\max } \mathrm{nm}$ : (MeOH): 260, 288 e 360. ES-MS (positive mode) $m / z 449[\mathrm{M}+\mathrm{H}]^{+} .{ }^{1} \mathrm{H}-\mathrm{NMR}$ (DMSO- $\left.d 6\right): \delta 7.41\left(1 \mathrm{H}, \mathrm{dd}, J=8.0,1.5 \mathrm{~Hz}, \mathrm{H}-6{ }^{\prime}\right), 7.40(1 \mathrm{H}, \mathrm{dd}, J=1.5 \mathrm{~Hz}$, H-2'), 6.88(1H, d, $\left.J=8.0 \mathrm{~Hz}, \mathrm{H}-5^{\prime}\right), 6.68(1 \mathrm{H}, \mathrm{s}, \mathrm{H}-8), 6.46(1 \mathrm{H}, \mathrm{s}, \mathrm{H}-3), 4.61(1 \mathrm{H}, \mathrm{d}, J=10.0 \mathrm{~Hz}$, $\mathrm{H}-1$ "-Glc), 4.00 (2H, dd, 9.0, 9.0, H-2"-Glc), 3.70 (1H, dd, 5.5, 6.0, H-6b"-Glc), 3.37 (1H, dd, 5.5, 6.0, H-6a"-Glc), 3.21 (2H, d, 9.0, H-3"-Glc), 3.16 (1H, m, H-5"-Glc), 3.12 (1H, m, H-4"-Glc). ${ }^{13} \mathrm{C}-\mathrm{NMR}$ (DMSO-d6):181.0 (C, C-4), 163.7 (C, C-2-7), 160.0 (C, C-5), 156.0 (C, C-9), 154.6 (C, C-4'), 146.0 (C, C-3'), $121.6\left(\mathrm{C}, \mathrm{C}-1^{\prime}\right), 119.0\left(\mathrm{CH}, \mathrm{C}-6^{\prime}\right), 115.0\left(\mathrm{CH}, \mathrm{C}-5^{\prime}\right), 113.8\left(\mathrm{CH}, \mathrm{C}-2^{\prime}\right), 109.0$ (C, C-6), 104.0 (C, C-10), 102.9 (CH, C-3), 93.5 (CH, C-8), 81.2 (CH, C-5"-Glc), 78.2 (CH, C-3"-Glc), 73.0 (CH, C-1"-Glc), 70.4 (CH, C-2"-Glc), 70.8 (CH, C-4"'-Glc), 61.8 ( $\left.\mathrm{CH}_{2}, \mathrm{C}-66^{\prime \prime}-\mathrm{Glc}\right)$.

7-Methoxyluteolin-6-C- $\beta$-D-glucopyranoside (2): UV $\lambda_{\max } \mathrm{nm}:(\mathrm{MeOH}): 254,270$ e $349 \mathrm{~nm}$. ES-MS (negative mode): $m / \mathrm{z} 461[\mathrm{M}-\mathrm{H}]^{-}, 371[\mathrm{M}-90-\mathrm{H}]^{-}, 341[\mathrm{M}-120-\mathrm{H}]^{-} .{ }^{1} \mathrm{H}-\mathrm{NMR}$ (DMSO-d6): $\delta 7.46\left(1 \mathrm{H}, \mathrm{d}, J=1.5 \mathrm{~Hz}, \mathrm{H}-2^{\prime}\right), 6.88\left(1 \mathrm{H}, \mathrm{d}, J=8.0 \mathrm{~Hz}, \mathrm{H}-5^{\prime}\right), 6.79(1 \mathrm{H}, \mathrm{s}, \mathrm{H}-8), 6.75(1 \mathrm{H}, \mathrm{s}, \mathrm{H}-3)$, $6.48\left(1 \mathrm{H}, \mathrm{dd}, J=8.0,1.5 \mathrm{~Hz}, \mathrm{H}-6^{\prime}\right), 3.89\left(3 \mathrm{H}, \mathrm{s}, 7-\mathrm{OCH}_{3}\right), 4.57\left(1 \mathrm{H}, \mathrm{d}, J=10.0 \mathrm{~Hz}, \mathrm{H}-1^{\prime \prime} \mathrm{Glc}\right), 3.48$ dd (1H, dd, 9.0, 9.0, H-4"'-Glc), 3.70 (1H, dd, 3.5, 12.0, H-6a"-Glc), 3.38 (1H, dd, 4.5, 12.0, H-6b"-Glc), 3.16 (1H, dd, 10.5, 9.5, H-3"-Glc), 3.18 (1H, m, H-5"-Glc), 3.12 (1H, 9.0, 7.5, H-2"'-Glc). ${ }^{13} \mathrm{C}-\mathrm{NMR}$ (DMSO-d6): 181.0 (C, C-4), 163.9 (C, C-2), 163.7 (C, C-7), 156.9 (C, C-9), 156.6 (C, C-5), 149.9 (C, C-4'), 145.9 (C, C-3'), $121.4\left(\mathrm{C}, \mathrm{C}^{\prime} 1^{\prime}\right), 118.3\left(\mathrm{CH}, \mathrm{C}-6^{\prime}\right), 115.6\left(\mathrm{CH}, \mathrm{C}-5^{\prime}\right), 112.8$ (CH, C-2'), 109.6 (C, C-6), 104.3 (C, C-10), 103.0 (CH, C-3), 90.5 (CH, C-8), 81.9 (CH, C-5"-Glc), 78.2 (CH, C-3"-Glc), 72.8 (CH, C-1"-Glc), 70.4 (CH, C-2"-Glc), 71.4 (CH, C-4"-Glc), $61.4\left(\mathrm{CH}_{2}, \mathrm{C}-6{ }^{\prime \prime} \mathrm{Glc}\right), 56.5\left(\mathrm{C}-7-\mathrm{OCH}_{3}\right)$. 
7-Methoxyluteolin-8-C- $\beta$-D-glucopyranoside (3): UV $\lambda_{\max } \mathrm{nm}:(\mathrm{MeOH}): 258,288$ e 356. ES-MS (negative mode) $m / z 461[\mathrm{M}-\mathrm{H}]^{-}, 371[\mathrm{M}-90-\mathrm{H}]^{-}, 341[\mathrm{M}-120-\mathrm{H}]^{-} .{ }^{1} \mathrm{H}-\mathrm{NMR}$ (DMSO-d6): $\delta 7.54$ $\left(1 \mathrm{H}, \mathrm{dd}, J=8.0,1.5 \mathrm{~Hz}, \mathrm{H}-6^{\prime}\right), 7.48\left(1 \mathrm{H}, \mathrm{d}, J=1.5 \mathrm{~Hz}, \mathrm{H}-2^{\prime}\right), 6.86\left(1 \mathrm{H}, \mathrm{d}, J=8.0 \mathrm{~Hz}, \mathrm{H}-5^{\prime}\right), 6.68(1 \mathrm{H}, \mathrm{s}$, H-3), 6.50 (1H, s, H-6), 3.87 (3H, s, 7-OCH $), 4.70$ (1H, d, J=10.0 Hz, H-1"-Glc), 3.82 (1H, dd, 9.0, 9.5, H-4"-Glc), 3.68 (1H, dd, 3.5, 12.0, H-6a"'-Glc), 3.40 (1H, dd, 8.0, 7.5, H-3"-Glc), 3.34 (1H, dd, 4.5, 12.0, H-6b"-Glc), 3.24 (1H, dd, 8.5, 9.0, H-2"-Glc), 3.14 (1H, m, H-5"-Glc). ${ }^{13} \mathrm{C}-\mathrm{NMR}$ (DMSO-d6):181.0 (C, C-4), 163.8 (C, C-7), 164.4 (C, C-2), 161.1 (C, C-5), 155.1 (C, C-9), 149.7 (C, C-4'), 145.8 (C, C-3'), $121.6\left(\mathrm{C}, \mathrm{C}-1^{\prime}\right), 119.1\left(\mathrm{CH}, \mathrm{C}-6^{\prime}\right), 115.4\left(\mathrm{CH}, \mathrm{C}-5^{\prime}\right), 113.9\left(\mathrm{CH}, \mathrm{C}-2^{\prime}\right), 104.3$ (C, C-8), 104.3 (C, C-10), 102.4 (CH, C-3), 94.9 (CH, C-6), 81.6 (CH, C-5"-Glc), 78.2 (CH, C-3"-Glc) 72.8 (CH, C-1"'-Glc), 70.2 (CH, C-4"'-Glc), 70.2 (CH, C-2"'-Glc), $61.4\left(\mathrm{CH}_{2}, \mathrm{C}-6\right.$ "'- $\left.^{\prime} \mathrm{Glc}\right), 56.5\left(\mathrm{C}-7-\mathrm{OCH}_{3}\right)$.

4'-Methoxyluteolin-6-C- $\beta$-D-glucopyranoside (4): UV $\lambda_{\max } \mathrm{nm}:(\mathrm{MeOH}): 243,271$ e 342. ES-MS (positive mode) $m / z 463[\mathrm{M}+\mathrm{H}]^{+} .{ }^{1} \mathrm{H}-\mathrm{NMR}$ (DMSO-d6): $\delta 7.40\left(1 \mathrm{H}, \mathrm{d}, J=1.5 \mathrm{~Hz}, \mathrm{H}-2^{\prime}\right), 7.38(1 \mathrm{H}, \mathrm{dd}$, $\left.J=8.0,1.5 \mathrm{~Hz}, \mathrm{H}-6^{\prime}\right), 6.88\left(1 \mathrm{H}, \mathrm{d}, J=8.0 \mathrm{~Hz}, \mathrm{H}-5^{\prime}\right), 6.68(1 \mathrm{H}, \mathrm{s}, \mathrm{H}-8), 6.46(1 \mathrm{H}, \mathrm{s}, \mathrm{H}-3), 3.89$ (3H, s, $\left.4^{\prime}-\mathrm{OCH}_{3}\right), 4.68\left(1 \mathrm{H}, \mathrm{dd}, J=12.0,2.5 \mathrm{~Hz}, \mathrm{H}-6 \mathrm{~b}^{\prime \prime}-\mathrm{Glc}\right), 4.61\left(1 \mathrm{H}, \mathrm{d}, J=10.0 \mathrm{~Hz}, \mathrm{H}-1^{\prime \prime}-\mathrm{Glc}\right), 4.36$ (1H, dd, $\left.J=12.0,5.0 \mathrm{~Hz}, \mathrm{H}-6 \mathrm{a}^{\prime \prime}-\mathrm{Glc}\right), 4.00\left(1 \mathrm{H}, \mathrm{dd}, J=9.0,9.0 \mathrm{~Hz}, \mathrm{H}-2^{\prime \prime}-\mathrm{Glc}\right), 3.21(1 \mathrm{H}, \mathrm{dd}, J=9.0,9.0 \mathrm{~Hz}$, H-3"-Glc), 3.18 (1H, dd, $\left.J=9.0,9.0 \mathrm{~Hz}, \mathrm{H}-4^{\prime \prime}-\mathrm{Glc}\right), 3.15$ (1H, m, H-5"-Glc), 3.23 (1H, dd, J = 9.0, 9.0 Hz, H-4 Ara), 3.21 (1H, dd, $J=9.0,9.0 \mathrm{~Hz}, \mathrm{H}-3 \mathrm{Ara}) .{ }^{13} \mathrm{C}-\mathrm{NMR}$ (DMSO-d6): 181.0 (C, C-4), 163.4 (C, C-7), 160.0 (C, C-5), 154.6 (C, C-4'), 156.0 (C, C-9), 146.0 (C, C-3'), 121.6 (C, C-1'), 118.2 (CH, C-6'), 113.0 (CH, C-2'), 115.0 (CH, C-5'), 109.0 (C, C-6), 104.0 (C, C-10), 103.0 (CH, C-3), 93.6 (CH, C-8), 81.0 (CH, C-5"-Glc), 78.6 (CH, C-3"-Glc) 73.3 (CH, C-1"'-Glc), 70.8 (CH, C-4"'-Glc), 70.5 (CH, C-2"'-Glc), $61.0\left(\mathrm{CH}_{2}, \mathrm{C}-6 "-\mathrm{Glc}\right), 56.0\left(\mathrm{C} 7-\mathrm{OCH}_{3}\right)$.

6-Hydroxy-7-methoxyluteolin (5): UV $\lambda_{\max } \mathrm{nm}$ : (MeOH): 254, 273, 348, ES-MS (negative mode) $m / z$ $315[\mathrm{M}-\mathrm{H}]^{-} .{ }^{1} \mathrm{H}-\mathrm{NMR}$ (DMSO-d6): $\delta 7.42\left(1 \mathrm{H}, \mathrm{dd}, J=1.5 \mathrm{~Hz}, \mathrm{H}-2^{\prime}\right), 7.41\left(1 \mathrm{H}, \mathrm{dd}, J=8.0,1.5 \mathrm{~Hz}, \mathrm{H}-6^{\prime}\right)$, $6.89\left(1 \mathrm{H}, \mathrm{d}, J=8.0 \mathrm{~Hz}, \mathrm{H}-5^{\prime}\right), 6.66(1 \mathrm{H}, \mathrm{s}, \mathrm{H}-3), 6.84(1 \mathrm{H}, \mathrm{s}, \mathrm{H}-8), 3.90\left(3 \mathrm{H}, \mathrm{s}, 4^{\prime}-\mathrm{OCH}_{3}\right) .{ }^{13} \mathrm{C}-\mathrm{NMR}$ (DMSO-d6):182.1 (C, C-4), 163.8 (C, C-2), 155.6 (C, C-7), 152.0 (C, C-9), 151.0 (C, C-5), 149.7 (C, C-4'), 145.8 (C, C-3'), 131.4 (C, C-6), $121.6\left(\mathrm{C}, \mathrm{C}-1^{\prime}\right), 119.0\left(\mathrm{CH}, \mathrm{C}-6^{\prime}\right), 115.6\left(\mathrm{CH}, \mathrm{C}-5^{\prime}\right), 113.4(\mathrm{CH}$, C-2'), 102.4 (CH, C-3), 104.1 (C, C-10), $91.0(\mathrm{CH}, \mathrm{C}-8), 56.0\left(\mathrm{C} 7-\mathrm{OCH}_{3}\right)$.

8-Carboxymethyl-1,5,6-trihydroxy-3-methoxyxanthone (6): UV $\lambda_{\max } \mathrm{nm}:(\mathrm{MeOH}): 204,252,292,325$. EI-MS (positive mode) $m / z 332[\mathrm{M}]^{+}, 333[\mathrm{M}+\mathrm{H}]^{+} 301\left[\mathrm{M}-\mathrm{OCH}_{3}\right]^{+}, 273\left[\mathrm{M}-\mathrm{COOCH}_{3}\right] .{ }^{1} \mathrm{H}-\mathrm{NMR}$ (DMSO-d6): $\delta 6.15(1 \mathrm{H}, \mathrm{d}, J=1.5 \mathrm{~Hz}, \mathrm{H}-2), 6.38(1 \mathrm{H}, \mathrm{d}, J=1.5 \mathrm{~Hz}, \mathrm{H}-4), 6.79(1 \mathrm{H}, \mathrm{s}, \mathrm{H}-7), 3.79$ $\left(3 \mathrm{H}, \mathrm{s}, 3-\mathrm{OCH}_{3}\right),{ }^{13} \mathrm{C}-\mathrm{NMR}$ (DMSO-d6): $\delta 164.8$ (C-1), 98.1 (C-2), $168.2(\mathrm{C}-3), 93.8(\mathrm{C}-4), 157.4$ (C-4a), 146.7 (C-4b), 134.8 (C-5), 151.0 (C-6), 111.8 (C-7), 124.9 (C-8), 111.2 (C-8a), 180.0 (C-9), 102.7 (C-9a), 171.4 (C-10), $56.4\left(\mathrm{C}-3-\mathrm{OCH}_{3}\right)$.

8-Carboxymethyl-1,3,5,6-tetrahydroxyxanthone (7): UV $\lambda_{\max } \mathrm{nm}:(\mathrm{MeOH}): 205,244,288,315,362$. EI-MS (positive mode) $m / z 346[\mathrm{M}]^{+}, 315[\mathrm{M}-\mathrm{OCH} 3]^{+}, 285[\mathrm{M}-2 \mathrm{OCH} 3+\mathrm{H}]^{+} .{ }^{1} \mathrm{H}-\mathrm{NMR}$ (DMSO-d6): $\delta 6.34(1 \mathrm{H}, \mathrm{d}, J=1.5 \mathrm{~Hz}, \mathrm{H}-2), 6.57(1 \mathrm{H}, \mathrm{d}, J=1.5 \mathrm{~Hz}, \mathrm{H}-4), 6.78(1 \mathrm{H}, \mathrm{s}, \mathrm{H}-7), 3.87$ (3H, s, 3-OCH3), ${ }^{13} \mathrm{C}-\mathrm{NMR}$ (DMSO-d6): $\delta 163.8$ (C-1), 97.6 (C-2), 166.4 (C-3), 93.4 (C-4), 157.8 (C-4a), 146.0 (C-4b), 135.1 (C-5), 150.0 (C-6), 112.4 (C-7), 124.5 (C-8), 110.9 (C-8a), 179.0 (C-9), 103.0 (C-9a), 170.0 (C-10), $56.0(\mathrm{C}-3-\mathrm{OCH} 3)$. 


\subsection{Antibacterial Activity and Minimum Bactericidal Concentration (MBC)}

MIC was determined by the broth microdilution method, according to the standard reference method [40]. Mueller-Hinton broth (MHB) cultures of the standard Gram-positive and Gram-negative bacterial strains incubated for $24 \mathrm{~h}$ at $37{ }^{\circ} \mathrm{C}$ were used: Staphylococcus aureus (ATCC 25923), Bacilus subtillis (ATCC 19659), Enterococcus faecalis (ATCC 29212), Escherichia coli (ATCC 25922), Pseudomonas aeruginosa (ATCC 27853), and Salmonella setubal (ATCC 19196). The extract and compounds were dissolved in $25 \%$ methanol and water. The initial concentration was $1,000 \mu \mathrm{g} / \mathrm{mL}$ for extract and $500 \mu \mathrm{g} / \mathrm{mL}$ for isolated compounds. These initial volumes were serially diluted two-fold to obtain concentration ranges of $7.8-1,000 \mu \mathrm{g} / \mathrm{mL}$ for the extract and $3.9-500 \mu \mathrm{g} / \mathrm{mL}$ for the isolated compounds. $100 \mu \mathrm{L}$ of each concentration was placed in a 96-well microplate containing $80 \mu \mathrm{L}$ of MHB and $20 \mu \mathrm{L}$ of inoculum standardized at $1.0 \times 10^{8} \mathrm{CFU} / \mathrm{mL}$ by adjusting the optical density to 0.1 at $620 \mathrm{~nm}$ (Pharmacia LKB-Ultrospec III spectrophotometer). Ciprofloxacin (Bayer HealthCare ${ }^{\circledR}$, concentration ranges of $0.5 \times 10^{-5}-35 \mu \mathrm{g} / \mathrm{mL}$ ) and $25 \%$ methanol and water were used as positive and negative controls. The plates were incubated at $37{ }^{\circ} \mathrm{C}$ for $24 \mathrm{~h}$. The assay was repeated three times. The MIC of samples was detected following addition $(50 \mu \mathrm{L})$ of resazurin solution $(0.2 \mathrm{mg} / \mathrm{mL})$ and incubated at $37^{\circ} \mathrm{C}$ for $30 \mathrm{~min}$. Growth of bacteria changes the blue dye to a pink color. The pink color indicates positive growth, while blue indicates growth inhibition. MIC was defined as the lowest sample concentration that prevented this change and inhibited bacterial growth. To determine MBC, a portion from each well showing antibacterial activity (MIC) was plated on Muller-Hinton agar (MHA) and incubated at $37{ }^{\circ} \mathrm{C}$ for $24 \mathrm{~h}$. The lowest concentration yielding no growth after this subculture was defined as the MBC [41].

\subsection{Antifungal Activity and Minimum Fungicidal Concentration (MFC)}

Antifungal activity and MIC were determined according to the standard reference method [42], evaluated against Candida albicans (ATCC 18804), C. krusei (ATCC 6258), C. parapsilosis (ATCC 22019), and C. tropicalis (ATCC 750). The extracts and compounds were dissolved in 25\% methanol and water. The initial concentration was $1,000 \mu \mathrm{g} / \mathrm{mL}$ for extract and $500 \mu \mathrm{g} / \mathrm{mL}$ for isolated compounds. These initial volume were diluted two-fold to obtain concentration ranges of $7.8-1,000 \mu \mathrm{g} / \mathrm{mL}$ for the extract and 3.9-500 $\mu \mathrm{g} / \mathrm{mL}$ for the isolated compounds. $100 \mu \mathrm{L}$ of each concentration was placed in a 96-well microplate containing $80 \mu \mathrm{L}$ of RPMI 1640 medium. Each well was inoculated with $20 \mu \mathrm{L}$ of suspension containing $2.5 \times 10^{3} \mathrm{CFU} / \mathrm{mL}$ of yeast by adjusting the optical density to $0.8-0.1$ at $625 \mathrm{~nm}$. The antifungal agent fluconazole (Pfizer ${ }^{\circledR}$, concentration ranges of $1-64 \mu \mathrm{g} / \mathrm{mL}$ ) and $25 \%$ methanol and water were included in the assays as positive and negative controls. The plates were incubated for $48 \mathrm{~h}$ at $37^{\circ} \mathrm{C}$. MIC was defined as the lowest sample concentration showing no visible fungal growth after incubation time. The MIC of samples was detected following addition $(50 \mu \mathrm{L})$ of $2.0 \%$ triphenyltetrazolium chloride (TTC). Growth of yeast changes the samples to a red color. Three replications were maintained. A portion from each well with antifungal activity was plated on Sabouroud agar for to determination of MFC. 


\subsection{Anti-Helicobacter pylori Activity}

Phenolic compounds were tested to detect anti-H. pylori (ATCC 43504) activity [43,44]. H. pylori was cultured in MHB (with $5 \%$ calf serum) at $37{ }^{\circ} \mathrm{C}$ for $3-5$ days in a microaerobic atmosphere (10\% $\mathrm{CO}_{2}$ and $98 \%$ humidity). Muller-Hinton agar (with 5\% calf serum) was used for susceptibility testing. Disks of $6 \mathrm{~mm}$ in diameter were punched from a sheet of Whatman filter paper, sterilized, and impregnated with $25 \mu \mathrm{L}$ of each sample $(31,25,62,5,125$ and $250 \mu \mathrm{g} / \mathrm{mL})$ or solvent alone. The bacterial inoculum was prepared and adjusted to $10^{8} \mathrm{CFU} / \mathrm{mL}$ (corresponding to $0.5 \mathrm{McFarland}$ standards). A sterile cotton swab was dipped into the standardized bacterial suspension and used to inoculate the Muller-Hinton agar plates. The plates were allowed to dry for 3-5 min. After that, all disks were placed in plates and maintained at a distance of at least $15 \mathrm{~mm}$ from the plate edges and sufficiently separated from each other to prevent overlapping of inhibition zones. A clarithromycin (Abbott $\left.{ }^{\circledR}\right)$ disk $(15 \mu \mathrm{g} / \mathrm{mL})$, and 25\% methanol and water solution-impregnated disks were used as controls. Fifteen minutes following placement of the disks, the plates of H. pylori were incubated at $37{ }^{\circ} \mathrm{C}$ for $3-5$ days in a microaerobic atmosphere $\left(10 \% \mathrm{CO}_{2}\right.$ and $98 \%$ humidity). They were then examined and the diameter of the inhibition zone measured.

\section{Conclusions}

The antimicrobial activity of methanolic extract of leaves from L. spiralis possibly results from the presence of both antibacterial and antifungal compounds. These findings could provide an important contribution in the search for new compounds with antimicrobial activity.

\section{Acknowledgements}

The authors gratefully acknowledge the financial support of Fundação de Amparo à Pesquisa do Estado de São Paulo (FAPESP), which provided a fellowship to F. Hilario and a project grant to L.C. Santos. Coordenação de Aperfeiçoamento de Pessoal de Nível Superior (CAPES) provided a fellowship to M.G.F. Araújo. We also thank Conselho Nacional de Desenvolvimento Científico e Tecnológico (CNPq) and Fundação para o Desenvolvimento da UNESP (FUNDUNESP).

\section{Conflict of Interest}

The authors declare that they have no conflict of interest.

\section{References and Notes}

1. Giulietti, A.M.; Amaral, M.C.E.; Bittrich, V. Phylogenetic analysis of inter-and infrageneric relationships of Leiothrix Ruhland (Eriocaulaceae). Kew Bull. 1995, 50, 55-71.

2. Coelho, R.G.; Batista, L.M.; Santos, L.C.; Souza-Brito, A.R.M.; Vilegas, W. Phytochemical study and antiulcerogenic activity of Syngonanthus bisulcatus (Eriocaulaceae). Rev. Bras. Cienc. Farm. 2006, 42, 413-417.

3. Hoehne, F.C. Outras monocotyledoneas tóxicas ou suspeitas. In Plantas e substâncias vegetais tóxicas e medicinais, Hoehne, F.C., Ed.; Graphicards: São Paulo/Rio de Janeiro, Brazil, 1939; p. 97. 
4. Santos, L.C.; Piacente, S.; Cosimo, P.; Montoro, P.; Vilegas V. Antioxidante de xantonas isoladas de espécies de Leiothrix (Eriocaulaceae). Rev. Braz. Farmacog. 2003, 13, 67-74.

5. Varanda, E.A.; Raddi, M.S.G.; Dias, F.L.P.; Araujo, M.C.S.; Gibran, S.C.A.; Takahashi, C.S.; Vilegas, W. Mutagenic and cytotoxic activity of an isocoumarin (paepalantine) isolated from Paepalanthus vellozioides. Teratog. Carc. Mut. 1997, 17, 85-95.

6. Tavares, D.C.; Varanda, E.A.; Andrade, F.P.D.; Vilegas, W.; Takahashi, C.S. Evaluation of the genotoxic potential of the isocoumarin paepalantine in in vivo and in vitro mammalian systems. J. Ethnopharmacol. 1999, 68, 115-120.

7. Batista, L.M.; Almeida, A.B.; Pietro, L.M.; Toma, W.; Calvo, T.R.; Vilegas, W.; Souza, A.R.B. Gastric antiulcer activity of Syngonanthus arthrotrichus Silveira. Biol. Pharm. Bull. 2004, 27, 328-332.

8. Sano, P.T. Actinocephalus (Koern.) Sano (Paepalanthus sect. Actinocephalus), a new genus of Eriocaulaceae, and other taxonomic and nomenclatural changes involving Paepalanthus Mart. Taxon 2004, 53, 99-107.

9. Dokkedal, A.L.; Salatino, A. Flavonoids of Brazilian species of Leiothrix (Eriocaulaceae). Biochem. Syst. Ecol. 1992, 20, 31-32.

10. Salatino, A.; Salatino, M.L.; Giulietti, A.M. Contents of soluble phenolic compounds of capitula of Eriocaulaceae. Quim. Nova 1990, 13, 289-292.

11. Santos, L.C.; Piacente, S.; Montoro, P.; Pizza, C.; Vilegas W. Atividade antioxidante de xantonas isoladas de espécies de Leiothix (Eriocaulaceae). Rev. Bras. Farmacog. 2003, 13, 67-74.

12. Vilegas, W.; Dokkedal, A.L.; Rastrelli, L.; Piacente, S.; Pizza, C. New naphtopyranone glycoside from Paepalanthus vellozioides and Paepalanthus latipes. J. Nat. Prod. 1999, 62, 746-749.

13. Santos, L.C.; Piacente, S.; Riccardis, F.D.; Eletto, A.M.; Pizza, C.; Vilegas, W. Xanthones and flavonoids from Leiothrix curvifolia and Leiothrix flavescens. Phytochemistry 2001, 56, 853-856.

14. Ricci, C.V.; Patricio, M.C.B.; Salatino, M.L.F.; Salatino, A.; Giulietti, A.M. Flavonoids of Syngonanthus Ruhl. (Eriocaulaceae): Taxonomic implications. Biochem. System. Ecol. 1996, 24, 577-583.

15. Elix, J.A.; Bennett, S.A. 6- $O$-methylarthothelin and 1,3,6-tri- $O$-methylarthothelin, two new xanthones from a Dimelaena lichen. Aust. J. Chem. 1990, 43, 1587-1590.

16. Tanahashi, T.; Takenaka, Y.; Ikuta, Y.; Tani, K.; Nagakura, N.; Hamada, N. Xanthones from the cultured lichen mycobionts of Pyrenula japonica and Pyrenula pseudobufonia. Phytochemistry 1999, 52, 401-405.

17. Holetz, F.B.; Pessini, G.L.; Sanches, N.R.; Cortez, D.A.G.; Nakamura, C.V.; Filho, B.P.D. Screening of some plants used in the Brazilian folk medicine for the treatment of infectious diseases. Mem. Inst. Oswaldo Cruz 2002, 97, 1027-1031.

18. Gibbons, S. Phytochemicals for bacterial resistance-strengths, weaknesses and opportunities. Planta Med. 2008, 74, 594-602.

19. Lee, Y.J.; Liu, H.Y.; Lin, Y.C.; Sun, K.L.; Chun, C.L.; Hsueh, P.R. Fluoroquinolone resistance of Pseudomonas aeruginosa isolates causing nosocomial infection is correlated with levofloxacin but not ciprofloxacin use. Int. J. Antimicrob. Agents 2010, 35, 261-264. 
20. Micek, S.T.; Lloyd, A.E.; Ritchie, D.J.; Reichley, R.M.; Fraser, V.J.; Kollef, M.H. Pseudomonas aeruginosa bloodstream infection: Importance of appropriate initial antimicrobial treatment. Antimicrob. Agents Chemother. 2005, 49, 1306-1311.

21. Fotie, J.; Bohle, S. Pharmacological and biological activities of xanthones. Curr. Med. Chem. 2006, 5, 15-31.

22. Marona, H.; Szkaradek, N.; Karczewska, E.; Trojanowska, D.; Budak, A.; Bober, P.; Przepirka, W.; Cegla, M.; Szneler, E. Antifungal and antibacterial activity of the newly synthesized 2-xanthone derivatives. Arch. Pharm. 2009, 342, 9-18.

23. Pinto, M.M.M.; Castanheiro, R. Natural Prenylated Xanthones: Chemistry and Biological Activities. In Natural Products: Chemistry, Biochemistry and Pharmacology; Brahmachari, G., Ed.; Narosa Publishing House PVT. LTD: New Dehli, India, 2009; Chapter 11, pp. 520-675.

24. Pinto, E.; Afonso, C.; Duarte, S.; Vale-Silva, L.; Costa, E.; Sousa, E.; Pinto, M. Antifungal activity of xanthones: Evaluation of their effect on ergosterol biosynthesis by High-Performance Liquid Chromatography. Chem. Biol. Drug Des. 2011, 77, 212-222.

25. Dambolena, J.S.; Zygadlo, J.A.; Rubinstein, H.R. Antifumonisin activity of natural phenolic compounds. A structure-property-activity relationship study. Int. J. Food Microbiol. 2011, 145, 140-146.

26. Fang, J.J.; Ye, G.; Chen, W.L.; Zhao, W.M. Antibacterial phenolic components from Eriocaulon buergerianum. Phytochemistry 2008, 69, 1279-1286.

27. Hernández, N.E.; Tereschuk, M.L.; Abdala, L.R. Antimicrobial activity of flavonoids in medicinal plants from Tafí del Valle (Tucumán, Argentina). J. Ethnopharmacol. 2000, 73, 317-322.

28. Kuete, V.; Ngameni, B.; Simo, C.C.F.; Tankeu, R.K.; Ngadjui, B.T.; Meyer, J.J.M.; Lall, N.; Kuiate, R.R. Antimicrobial activity of the crude extracts and compounds from Ficus chlamydocarpa and Ficus cordata (Moraceae). J. Ethnopharmacol. 2008, 120, 17-24.

29. Mbaveng, A.T.; Ngameni, B.; Kuete, V.; Simo, I.K.; Ambassa, T.; Roy, R.; Bezabih, M.; Etoa, F.X.; Ngadjui, B.T.; Abegaz, B.M.; et al. Antimicrobial activity of the crude extracts and five flavonoids from the twigs of Dorstenia barteri (Moraceae). J. Ethnopharmacol. 2008, 116, 483-489.

30. Cushnie, T.P.T.; Lamb, A.J. Recent advances in understanding the antibacterial properties of flavonoids. Int. J. Antimicrob. Agents 2011, 38, 99-107.

31. Cushnie, T.P.T.; Lamb, A.J. Antimicrobial activity of flavonoids. Int. J. Antimicrob. Agents 2005, 26, 343-356.

32. da Silva, M.A.; Cardoso, C.A.; Vilegas, W.; dos Santos, L.C. High-performance liquid chromatographic quantification of flavonoids in Eriocaulaceae species and their antimicrobial activity. Molecules 2009, 14, 4644-4654.

33. Moraes, T.M.; Silva, M.A.; Rodrigues, C.M.; Santos, L.C.; Sannomiy, M.; Rocha, L.R.M.; Brito, A.R.M.S.; Bauab, T.M.; Vilegas, W.; Hiruma-Lima, C.A. Antioxidant, Antisecretory and Gastroprotective Activities from Leiothrix Flavescens. In Comprehensive Bioactive Natural Products. Efficacy, Safety \& Clinical Evaluation I., Gupta, V.K., Ed.; Studium Press LLC: Houston, TX, USA, 2010; Volume 2, Chapter 13, p. 265.

34. Rinaldo, D.; Rodrigues, C.M.; Rodrigues, J.; Sannomiya, M.; dos Santos, L.C.; Vilegas, W. New flavone from the leaves of Neea theifera (Nyctaginaceae). J. Braz. Chem. Soc. 2007, 18, 1132-1135. 
35. Miyake, Y.; Yamamoto, K.; Morimitsu, Y.; Osawa, T. Isolation of $C$-glucosylflavone from lemon peel and antioxidative activity of flavonoid compounds in lemon fruit. J. Agric. Food Chem. 1997, 45, 4619-4623.

36. Bai, N.; He, K.; Zhou, Z.; Lai, C.S.; Zhang, L.; Quan, Z.; Shao, X.; Pan, M.H.; Ho, C.T. Flavonoids from Rabdosia rubescens exert anti-inflammatory and growth inhibitory effect against human leukemia HL-60 cells. Food Chem. 2010, 122, 831-835.

37. Bjoroy, O.; Rayyan, S.; Fossen, T.; Kalberg, K.; Andersen, O.M. C-glycosylanthocyanidins synthesized from C-glycosylflavones. Phytochemistry 2009, 70, 278-287.

38. Wang, R.F.; Yang, X.W.; Ma, C.M.; Liu, H.Y.; Shang, M.Y.; Zhang, Q.Y.; Cai, S.Q.; Park, J.H. Trollioside, a new compound from the flowers of Trollius chinensis. J. Asian Nat. Prod. Res. 2004, 6, 139-144.

39. Ramesh, P.; Nair, A.G.R.; Subramanian, S.S. Flavone glycosides of Vitex trifolia. Fitoterapia 1986, 57, 282-283.

40. Clinical and Laboratory Standards Institute document M7-A8. Methods for Dilution Antimicrobial Susceptibility Tests for Bacteria that Grow Aerobically; Approved Standard, 7th ed.; Clinical and Laboratory Standards Institute: Wayne, PA, USA, 2009.

41. Kuete, V.; Nana, F.; Ngameni, B.; Mbaveng, A.T.; Keumedjio, F.; Ngadjui, B.T. Antimicrobial activity of the crude extract, fractions and compounds from stem bark of Ficus ovata (Moraceae). J. Ethnopharmacol. 2009, 124, 556-561.

42. Clinical and Laboratory Standards Institute document M27-A3. Reference Method for Broth Dilution Antifungal Susceptibility Testing of Yeasts; Approved Standard, 3th ed.; Clinical and Laboratory Standards Institute: Wayne, PA, USA, 2008, Volume 22.

43. Mégraud, F.; Lehours, P. Helicobacter pylori detection and antimicrobial susceptibility testing. Clin. Microbiol. Rev. 2007, 20, 280-322.

44. Clinical and Laboratory Standards Institute document M02-A10. Performance Standards for Antimicrobial Disk Susceptibility Tests; Approved Standard, 10th ed.; Clinical and Laboratory Standards Institute: Wayne, PA, USA, 2009.

Sample Availability: Not available.

(C) 2011 by the authors; licensee MDPI, Basel, Switzerland. This article is an open access article distributed under the terms and conditions of the Creative Commons Attribution license (http://creativecommons.org/licenses/by/3.0/). 